\title{
The effect of CS-induced fear on a partially extinguished CER'
}

\section{VERNON L. QUINSEY and JOHN J. B. AYRES, University of Massachusetts, Amherst, Mass. 01002}

The experiment was designed to determine whether (a) fear or (b) some nonmotivational variable produces shock-induced facilitation of the CER. After partial extinction of a CER to a light $C S$, rats received seven tone presentations before another extinction session. For $12 \mathrm{Ss}$ the tone had predicted shock but for 12 others had not. Although control groups showed that all Ss feared the tone presentations, the tones did not facilitate the CER to the light.

In a recent conditioned suppression or CER study, Hoffman (1965) gave tone-shock pairings to pigeons as they responded on a VI, food-supported schedule. Training continued until there was complete conditioned suppression to the CS but no suppression of baseline key pecking. Shockless stimulus generalization tests were then given until only slight suppression was elicited by the CS. Generalization tests were continued with interspersed time outs (periods of darkness). A shock was given in each time-out, and the next tone CS was presented more than $6 \mathrm{~min}$ after the time-out terminated. Hoffman found that the introduction of free shock had little effect on the baseline response rate but produced a large and immediate recovery of suppression to all the tones, including the original CS. Some CER facilitation remained on the day following the last session in which shock was given. From his data Hoffman inferred that an increase in emotionality facilitates a weakened CER. However, these data can also be explained in strictly associative terms. That is, introduction of the free shock may have merely reinstated stimulus conditions present during CER acquisition. To the extent that these conditions gained control over the CER during acquisition, their reinstatement in extinction should have increased the amount of CS-elicited conditioned suppression.

The present experiment was designed to determine the effects of fear induction per se on a partially extinguished CER. An attempt was made to increase the emotionality of one group during CER extinction without reintroducing the US used in the original CER conditioning and without reinstating acquisition cues for this "fear induction" group that were not also reinstated for a control group that did not receive fear induction. This was accomplished by presenting a stimulus in extinction that both groups had experienced in acquisition but that had been paired with shock for only the "fear induction" group.

\section{SUBJECTS}

Forty-eight experimentally naive, male, Sprague Dawley rats, 80-days-old upon their arrival at the laboratory, were obtained from Badger Research, Madison, Wisconsin. The Ss were fed ad lib for five days and then, seven days prior to the experiment, were reduced to and maintained at $80 \%$ of their free feeding body weight. During the experiment, Ss were weighed before, and fed 5 to $10 \mathrm{~min}$ after each session.

\section{APPARATUS}

Six Model C Gerbrands' operant conditioning chambers with left side dipper feeders (Model B) were housed in picnic coolers equipped with ventilating fans. Continuous white noise of $87-\mathrm{dB}$ intensity was presented through a speaker mounted on the lid of each chamber. One of the CSs was a $1000-\mathrm{Hz}$, 80-dB tone produced by a General Radio tone generator and presented through the white noise speaker but never simultaneously with the white noise. Both measures of sound pressure level were made inside the testing chamber when the fans were on. A second CS was provided by a change in light intensity effected by switching the voltage passing through a $6-\mathrm{W}, 24 \mathrm{~V}$ bulb from $6.5 \mathrm{~V}$ to $26 \mathrm{~V}$. The bulb was located in a 1 -in. white jewel pilot lamp located over the externally mounted food tray. Scrambled shocks were provided by six Grason-Stadler shock sources (Model E1064GS). Standard programming equipment controlled events in the experimental chambers from an adjacent room.

A $16 \%$ sucrose solution, prepared daily with tap water, was used as reward for dipper licking which was monitored by Grason-Stadler Model E4690A drinkometers and cumulated periodically for each $S$ on print out counters. Unless otherwise noted, the dipper was in the up position and available to $S$ for the first $4 \mathrm{sec}$ in every 5 -sec period throughout each session.

\section{PROCEDURE}

The experiment consisted of eight daily sessions: (a) rate stabilization, (b) habituation to the tone CS, (c) habituation to the light CS, (d) pseudoconditioning or CER conditioning with the tone CS, (e) CER conditioning with the light $\mathrm{CS}$, (f) recovery of lick rate, (g) partial extinction of the light CS, and $(h)$ free tones and testing with either the light or the tone. Ss were arbitrarily assigned to four equal groups in a 2 by 2 factorial using the variables conditioning or pseudoconditioning with the tone (CT or PCT) and (after presentation of free tones) testing with the light or with the tone (L or T). The groups employed are designated CT-T, CT-L, PCT-T, and PCT-L, where the first grouping describes the acquisition condition in Session $d$ and the second describes the testing condition in Session $h$. Suppression to the tone was measured in testing for half the Ss in order to determine the extent to which the tones still elicited fear after the free tones had been presented. Suppression in this test would indicate that the free tones had themselves induced emotionality.

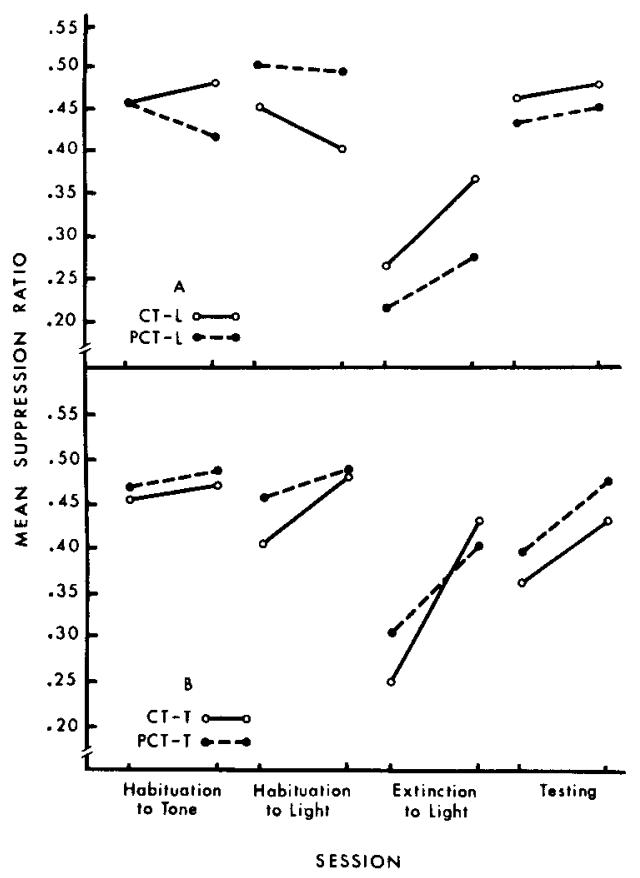

Fig. 1. Mean suppression ratios for each CS presentation of: (A) Groups CT-L and PCT-L, and (B) Groups CT-T and PCT-T. 
of change in this phenomenon over the four replications analyzed as well as those earlier in training.

\section{REFERENCES}

ISAAC, W., \& KENDALL, N. P. Sensory stimulation and timing beh avior. Psychonomic Science, 1967, 8, 41-42.
WORTHINGTON, C. S. Paradoxical illumination effects upon the behavior of two species of Cebid monkeys. Unpublished dissertation, Emory University, 1968.

\section{NOTES}

1. This work was supported by Research Grant MH 04539 from the National Institute of Mental Health.

2. Present address: University of Georgia, Athens Ga. 30601

(Continued from page 242)

Rate stabilization, habituation, recovery, and extinction sessions were all of 15-min duration. CS presentations of 1-min occurred in the eighth and twelfth minute of the habituation and extinction sessions. The rate stabilization and recovery sessions contained no CS presentations.

In Session d, Groups CT-T and CT-L received a 43-min, 20-sec CER training session consisting of 20 tone-shock pairings with a variable intertrial interval (VITI) of $2 \mathrm{~min}$. The tone CS duration was $10 \mathrm{sec}$, and the US, presented in the final second of the $\mathrm{CS}$, was a $2-\mathrm{mA}, 1-\mathrm{sec}$ shock. The two pseudoconditioning groups (PCT-T and PCT-L) were treated similarly to the CT-T and CT-L groups except that the tone and shock occurred randomly with respect to each other. Conditioning and pseudoconditioning occurred with the dipper inaccessible to Ss.

In Session e, 10 fear-conditioning trials were given to all Ss during a 22-min, 40-sec session with the dipper down. Trials were separated by a VITI of $2-\mathrm{min}$ and consisted of a $15-\mathrm{sec}$ change in light intensity coterminating with a 1-mA shock of 1 -sec duration. Ss were left in the experimental chambers for $15 \mathrm{~min}$ after the end of the session; no events were programmed during this period.

During testing, Ss received seven 10 -sec tones with a VITI of 2-min in a 15-min, 10-sec period in which the dipper was inaccessible. A 15-min licking session followed in which a 60-sec CS occurred following the seventh and eleventh minute. The CS was the tone for the CT-T and PCT-T Ss and the light for the CT-L and PCT-L Ss.

The data were analyzed in the form of suppression ratios, $D /(B+D)$, where $D$ represents the number of responses during the CS and $B$ the number of responses in an equal period of time immediately preceding it.

\section{RESULTS}

Data from the groups tested with the tone suggested that the free tones given prior to testing did induce emotionality (see Fig. 1, Panel B). Both the CT-T and the PCT-T groups suppressed more to the first $\mathrm{CS}$ in testing than to the last $C S$ in tone-habituation $(\mathrm{F}=44.10, \quad \mathrm{df}=1 / 22, \quad \mathrm{p}<.001, \quad$ and $\mathrm{F}=24.3, \mathrm{df}=1 / 22, \mathrm{p}<.001$ for CT-T and PCT-T, respectively). But surprisingly, these two groups did not differ in suppression to the first tone in testing $(F<1.0)$. Because the PCT-T and CT-T groups feared the tone equally, the free tones should not have differentially affected suppression to the light in testing for the CT-L and PCT-L groups. This prediction is confirmed by the nonsignificant difference $(F<1.0)$ between these groups on suppression to the first light CS following the free tones (see Panel A).

Since the free tones induced emotionality in both the CT-L and PCT-L groups, the motivational hypothesis requires that these groups show more suppression to the first CS presentation in testing than to the last presentation of the same CS in the extinction session. Both the CT-L and the PCT-L groups licked more during the first CS in testing than in the last $C S$ in extinction $(F=8.83, \mathrm{df}=1 / 44, p=.005$, and
$\mathrm{F}=21.8, \mathrm{df}=1 / 44, \mathrm{p}<.00 \mathrm{l}$, respectively), contradicting the motivational interpretation of CER enhancement.

All groups suppressed to the light in the extinction session and showed a marked decrement in suppression over extinction trials. Both of these effects were highly reliable. Finally, Ss receiving the conditioning procedure with the tone did not suppress more to the light in extinction than Ss receiving the pseudoconditioning procedure with the tone $(F=1.0)$; that is, no transfer was observed. However, in view of the fact that the CT-T and PCT-T groups could not be distinguished later on the basis of their suppression to the tone, this result is difficult to interpret.

\section{DISCUSSION}

The major finding of this research was that emotionality produced by a tone CS did not enhance suppression to another CS as was expected on the basis of the emotionality hypothesis. Because of the many procedural differences between Hoffman's free-shock study and the present one, it cannot be determined whether the discrepant results are due to the method of inducing emotionality or to some other factor. In this regard, Quinsey \& Ayres (1969), using a paradigm similar to that used here, were unable to enhance a partially extinguished CER with free shock but succeeded in arresting CER extinction, thereby enhancing the CER relative to that of a spontaneous recovery control. We tentatively conclude, therfore, that fear induction is not sufficient to enhance a partially extinguished CER or to stop its further extinction.

It was observed earlier that in Hoffman's experiment both a motivational and an associative interpretation lead to the same prediction. This is not the case in the present study because the tone-shock pairings occurred a full day before the light-shock pairings. It is unlikely that the tones were part of the stimulus context in which the CER to the light was learned. In the present study, the associative hypothesis predicts, therefore, that the free-tone presentations should not have enhanced suppression to the light.

The apparent pseudoconditioning of the PCT-T Ss may be due to remote associations facilitated by the relatively large number of high intensity shocks they received. Quinsey \& Ayres (1969), using a similar procedure but with fewer shocks of lower intensity, failed to demonstrate pseudoconditioning.

\section{REFERENCES}

HOFFMAN, H. S. The stimulus generalization of conditioned suppression. In D. 1. Mostofsky (Ed.), Stimulus generalization. Stanford: Stanford University Press, 1965. Pp. 356-372.

QUINSEY, V. L., \& AYRES, J. J. B. Shock-induced facilitation of a partially extinguished CER. Psychonomic Science, 1969, 14, 213-214. NOTE

1. This study represents part of Vernon L. Quinsey's M.S. thesis. It was done under the direction of John J. B. Ayres and was partially supported by a grant to John J. B. Ayres from the Faculty Research Council of the University of Massachusetts. 